\title{
A case of solitary breast metastasis from malignant melanoma of the nasal cavity
}

\author{
SATORU TANAKA, NAYUKO SATO, HIROYA FUJIOKA, YUKO TAKAHASHI, \\ KOSEI KIMURA, MITSUHIKO IWAMOTO and KAZUHISA UCHIYAMA
}

\begin{abstract}
Section of Breast and Endocrine Surgery, Department of General and Gastroenterological Surgery, Osaka Medical College, Takatsuki, Osaka 569-8686, Japan
\end{abstract}

Received May 21, 2012; Accepted August 16, 2012

DOI: $10.3892 / 01.2012 .871$

\begin{abstract}
Metastatic breast tumors are rare; however, malignant melanoma is one of the primary tumors most commonly reported to metastasize to the breast. Among these, the primary tumors typically associated with metastasis to the breast are cutaneous melanomas. We present, herein, a very rare case of solitary metastasis to the left breast from malignant melanoma of the nasal cavity, which represents less than $1 \%$ of all malignant melanomas. The patient, a 78-year-old woman, was treated using a combination of surgery and radiotherapy. In the absence of therapeutic efficacy, disease progression was very rapid. No previous studies have described malignant melanoma of the nasal cavity with solitary breast metastasis. This case report aims to increase awareness of the need to establish treatment strategies based on an understanding of the etiology and pathophysiology.
\end{abstract}

\section{Introduction}

Primary breast cancer is the most common malignancy among females worldwide; however, cancer tumors metastasizing to the breast are fairly rare. The most common cause is spread from a primary cancer in the contralateral breast (1). Other extramammary tumors often metastasizing to the breast include lymphoma, malignant melanoma and lung cancer (2).

Although malignant melanoma involves the skin in the vast majority of cases, lesions affecting the mucosa of the head and neck are rare. Involvement of the nasal cavity is even less common, accounting for less than $1 \%$ of all malignant melanomas (3). The clinical features of malignant melanoma of the nasal cavity are non-specific and show poor prognosis due to

Correspondence to: Dr Satoru Tanaka, Section of Breast and Endocrine Surgery, Department of General and Gastroenterological Surgery, Osaka Medical College, 2-7 Daigaku-machi, Takatsuki, Osaka 569-8686, Japan

E-mail: sur112@poh.osaka-med.ac.jp

Key words: malignant melanoma, breast, metastasis local recurrence, nodal involvement and distant metastasis (4). Among malignant melanomas with metastasis to the breast, cutaneous melanomas are the most common (5).

We recently treated a patient with a solitary metastatic breast tumor from a malignant melanoma of the nasal cavity. We present this rare case and discuss the relevant literature herein.

\section{Case report}

In November 2010, a 78-year-old female presented to the Department of Otorhinolaryngology at Osaka Medical College Hospital complaining of occasional epistaxis over the course of 6 weeks. Rhinoscopy revealed a brown-black tumor in the left nasal cavity. Head and neck computed tomography (CT) revealed a tumor in the left nasal cavity with invasion to the orbital cavity (Fig. 1A). Biopsy of the tumor led to a histological diagnosis of malignant melanoma. On pathological inspection, a hypervascular, pigmented lesion comprising round to oval-shaped tumor cells was revealed, and a final diagnosis of malignant melanoma was confirmed by immunohistochemical examination for HMB-45, a melanoma marker (Fig. 2A and B). During the investigation of the nasal cavity tumor, the patient had become aware of a growing lump in the upper region of the left breast and was referred to our department in December 2010. Physical examination revealed a well-defined, elastic, soft, immobile tumor in the upper region of the left breast, $4 \mathrm{~cm}$ in diameter. Mammography revealed an almost oval-shaped, microlobulated tumor in the left upper breast (Fig. 3). Ultrasonography revealed a heterogeneous mass with unclear margins. Fine needle aspiration cytology from the breast tumor was suggestive of metastatic melanoma, revealing cells similar in character to those of the tumor in the nasal cavity. No evidence of metastasis besides that to the left breast was found on positron emission tomography-CT. Radical resection of both the primary tumor and metastasis was proposed. The patient declined both radical surgery of the nasal cavity, in a maximally invasive operation requiring ophthalmectomy, and palliative surgery including systemic chemotherapy; however, breast surgery was accepted and a left mastectomy with lower axillary lymph node dissection, involving partial resection of the pectoralis major muscle, was performed as 
A
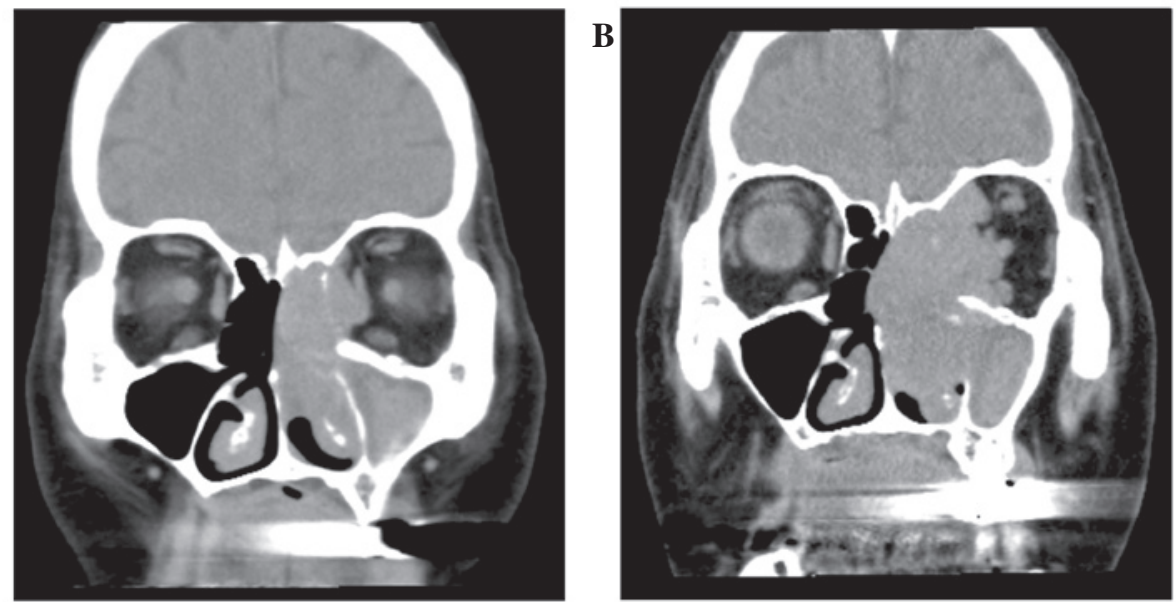

Figure 1. (A) Computed tomography of the head and neck, showing a mass in the left nasal cavity and fluid collection in the paranasal sinus, suggesting paranasal sinusitis. The tumor has invaded to the left orbital cavity. (B) Regrowth of the tumor following shrinkage with heavy ion radiotherapy.
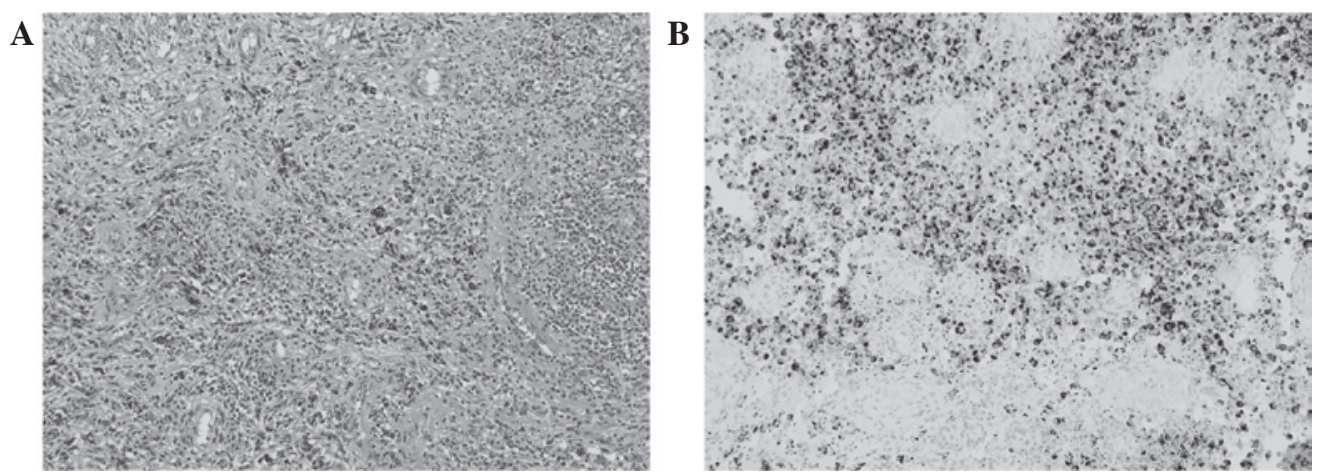

Figure 2. Malignant melanoma. (A) Infiltrating neoplastic cells containing brown melanin pigment. Abundant angiogenesis is evident within the submucosa of the nasal cavity. H\&E; magnification, x100. (B) High-intensity positive immunostaining for HMB-45 within tumor cells (magnification, x100). H\&E, hematoxylin and eosin.

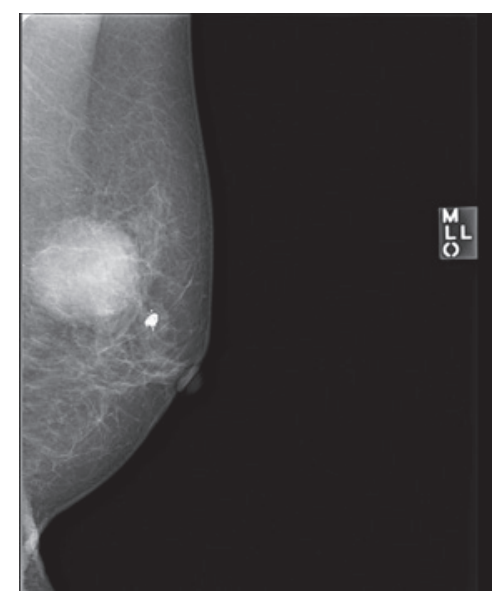

Figure 3. Mammography showing an almost oval-shaped, microlobulated tumor, assessed as a suspicious abnormality.

a result of suspected muscle invasion (Fig. 4). Although the dissected axillary lymph nodes were swollen, no metastasis of malignant melanoma was apparent under microscopy. For treatment of the primary site, heavy ion radiotherapy was performed in accordance with the wishes of the patient.

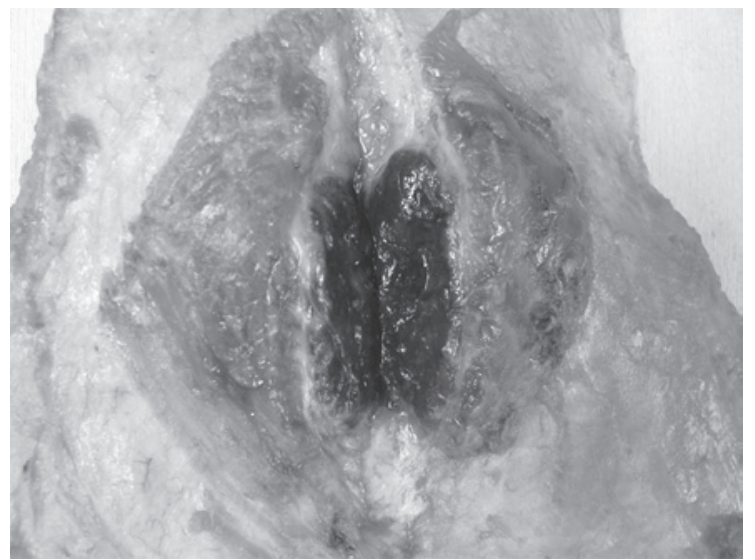

Figure 4. Macroscopic evaluation of the resected breast, revealing a brown-black tumor with a soft consistency.

The tumor initially reduced in size by $30 \%$ over a 2 -month period; however, it showed regrowth during the month of radiotherapy (Fig. 1B). The disease subsequently progressed very rapidly. Anticancer therapy was eventually suspended 3 months after the initial radiotherapy and only palliative 
care was administered. Finally, the patient was admitted into a hospice in August 2011.

\section{Discussion}

The clinically observed incidence of metastatic breast tumors was $0.5-1.2 \%$ in all breast neoplasms and $6 \%$ in an autopsy series (6). Georgiannos et al reviewed the clinical data for secondary tumors in the breast, revealing a frequency of $3.2 \%$, with the majority representing metastasis from a contralateral breast cancer (7).

Characteristics of metastatic breast tumors usually include presence in the superficial tissues and well-defined multinodular masses in the upper outer quadrant of the breast. The absence of calcification on radiological examination, such as mammography, is an additional suggestive feature (8). In the present case, a single breast tumor was present in the upper middle quadrant of the deeper mammary gland, therefore differentiation from primary breast neoplasm was required.

Malignant melanomas account for only $2-8 \%$ of all cancers arising in the nasal cavity, and mucosal melanoma of the nasal cavity is rarely encountered $(<1 \%$ of all malignant melanomas) (9). Given the rarity of this disease, no universally accepted staging system has been devised. The most fundamental treatment is wide resection of the primary site. Radiotherapy combined with surgery is recommended in cases of local recurrence or incomplete lesion removal; however, the majority of cases are resistant to radiotherapy, as in the present case. In addition, few standard systemic chemotherapies are regarded as effective. This disease is aggressive and distant metastases to the liver, lungs and brain, and regional metastases to subcutaneous tissues are the major causes of mortality in the majority of cases (10). In our institute, the 5-year survival rate for malignant melanoma of the nasal cavity is $36 \%$. In addition, the 2-year survival rate of patients with distant metastases is only $13 \%$, compared to $100 \%$ in the absence of distant metastases. Early detection and diagnosis with appropriate treatment should therefore be emphasized.

Metastases from cutaneous malignant melanoma represent the majority of cases of melanoma involving the breast with the most common primary sites associated with breast involvement on the arms and trunk (5). The mechanism of breast involvement in these cutaneous malignant melanomas may involve direct lymphatic and/or vascular drainage routes from the primary site to the breast. In the present case, breast metastasis was suggested to have occurred hematogenously, given that the primary tumor showed a rich blood supply and no axillary lymph node involvement was detected. As another mechanism, a hormonally-based association with the progression of melanoma has been suspected. Although the influence of estrogen in the development and progression of melanoma has been controversial, epidemiological evidence implicating estrogens in the etiology of melanoma has been accumulating (11). Estrogen receptors have been detected in certain melanoma cells, although at low levels and infrequently (12). The peak incidence age of melanoma among females coincides with the perimenopausal age. In a review of 15 patients with breast metastasis from cutaneous melanomas, the majority of patients (93\%) were premenopausal women with a mean age of 39 years (5). In another retrospective review of 27 patients with mammary metastasis from malignant melanoma, including cutaneous melanoma, $70 \%$ of patients were premenopausal (13).

In terms of the treatment for metastatic melanoma, insufficient results have been obtained using systemic chemotherapies, with response rates below $20 \%$ (14). The epidemiological and clinical evidence of melanoma potentially being an estrogendependent tumor suggests the possible efficacy of adding hormonal therapy to chemotherapy; however, single-agent hormonal therapy is minimally active. A preliminary report in which a high response rate was acquired using chemotherapy concurrent with tamoxifen has attracted attention (15). In addition, in a case series on malignant melanomas of the nasal cavity, 3 patients without distant metastases who responded to tamoxifen concurrent with chemotherapy were reported in 1997 (16). While a meta-analysis of randomized controlled trials did not demonstrate any significant improvement in the overall response rate, complete response rate or survival rate when tamoxifen was administered along with chemotherapy regimens for patients with metastatic melanoma; overall response rates tended to favor the combined regimens (17). In previous clinical trials and practices, tamoxifen, which was originally used as a target therapy to treat estrogen receptor-positive breast cancer patients, has been administered irrespective of the estrogen receptor status of tumor cells, therefore estrogen receptor-negative cases would have received no benefit from this hormonal therapy. In the present case, immunohistochemical staining for estrogen receptors yielded negative results (data not shown); however, hormonal therapy may be an option for patients with estrogen receptorpositive tumor cells, as this therapy is less toxic than chemo- or radiotherapy.

To the best of our knowledge, no previous reports in the English literature have described malignant melanoma of the nasal cavity with solitary breast metastasis. This case was unusual not only in that the primary site was the nasal cavity, but also because the solitary metastasis to the breast occurred in a postmenopausal woman. At present, radical surgery remains the only fundamental therapy; however, the establishment of treatment strategies based on a comprehensive understanding of both etiology and pathophysiology is needed for rare cases such as this.

\section{References}

1. McPherson K, Steel CM and Dixon JM: ABC of breast diseases. Breast cancer-epidemiology, risk factors, and genetics. BMJ 321: 624-628, 2000

2. Akçay MN: Metastatic disease in the breast. Breast 11: 526-528, 2002.

3. Khanna R, Srivastava RN and Agarwal A: Primary malignant melanoma of the nasal cavity. Ear Nose Throat J 69: 654-655, 1990.

4. Huang SF, Liao CT, Kan CR and Chen IH: Primary mucosal melanoma of the nasal cavity and paranasal sinuses: 12 years of experience. J Otolaryngol 36: 124-129, 2007.

5. Arora R and Robinson WA: Breast metastases from malignant melanoma. J Surg Oncol 50: 27-29, 1992.

6. Oksüzoğlu B, Abali H, Güler N, Baltali E and Ozișik Y: Metastasis to the breast from nonmammarian solid neoplasms: a report of five cases. Med Oncol 20: 295-300, 2003.

7. Georgiannos SN, Chin J, Goode AW and Sheaff M: Secondary neoplasms of the breast: a survey of the 20th Century. Cancer 92: 2259-2266, 2001 
8. Hajdu SI and Urban JA: Cancers metastatic to the breast. Cancer 29: $1691-1696,1972$

9. Kung B, Deschenes GR, Keane W, Cunnane M, Jacob-Ampuero MP and Rosen M: Paranasal sinus melanoma masquerading as chronic sinusitis and nasal polyposis. Ear Nose Throat J 86: 561-564, 2007.

10. Blatchford SJ, Koopmann CF Jr and Coulthard SW: Mucosal melanoma of the head and neck. Laryngoscope 96: 929-934, 1986.

11. Miller JG and Mac Neil S: Gender and cutaneous melanoma. $\mathrm{Br}$ J Dermatol 136: 657-665, 1997.

12. Lee YN: Better prognosis of many cancers in female: a phenomenon not explained by study of steroid receptors. J Surg Oncol 25: 255-262, 1984.

13. Ravdel L, Robinson WA, Lewis K and Gonzalez R: Metastatic melanoma in the breast: a report of 27 cases. J Surg Oncol 94: 101-104, 2006.
14. Atkins MB, Buzaid AC and Houghton AN: Systemic chemotherapy and biochemotherapy. In: Cutaneous Melanoma. Balch CM, Houghton A, Sober A and Soong S (eds). 4th edition. Quality Medical Publishing, St. Louis, pp589-604, 2003.

15. Del Prete SA, Maurer LH, O'Donnell J, Forcier RJ and LeMarbre P: Combination chemotherapy with cisplatin, carmustine, dacarbazine, and tamoxifen in metastatic melanoma. Cancer Treat Rep 68: 1403-1405, 1984.

16. Lens MB, Reiman T and Husain AF: Use of tamoxifen in the treatment of malignant melanoma. Cancer 95: 1355-1361, 2003.

17. Seo W, Ogasawara H and Sakagami M: Chemohormonal therapy for malignant melanomas of the nasal and paranasal mucosa. Rhinology 35: 19-21, 1997. 